INPLASY

PROTOCOL

To cite: Ni et al. Drug-Related

Problems of Patients in

Primary Heath Care

Institutions: a Systematic

Review. Inplasy protocol

202160081. doi:

10.37766/inplasy2021.6.0081

Received: 22 June 2021

Published: 22 June 2021

Corresponding author:

Lingli Zhang

zhanglingli@scu.edu.cn

Author Affiliation:

West China Second

University Hospital, Sichuan

University, China.

Support:

Youth Program of National

Natu.

Review Stage at time of this submission: Formal

screening of search results against eligibility criteria.

Conflicts of interest:

None declared.

\section{Drug-Related Problems of Patients in Primary Heath Care Institutions: a Systematic Review}

\author{
Ni, X1; Yang, C2; Bai, Y3; Hu, Z4; Zhang, L5.
}

Review question / Objective: This systematic review aimed to comprehensively review the characteristics of DRPs for patients in primary health care institutions, which might be helpful to find effective strategies to identify, prevent and intervene with DRPs in the future.

Condition being studied: The condition of DRPs in primary health care institutions should be paid more attention, but there is a lack of systematic review to comprehensively analyze the characteristics of DRPs for patients in primary health care institutions.

Information sources: 3 English databases (PubMed, The Cochrane library, EMbase) and 4 Chinese databases (CNKI, CBM, VIP, Wanfang)electronic databases, contact with authors, trial registers, or grey literature3 English databases (PubMed, The Cochrane library, EMbase) and 4 Chinese databases (CNKI, CBM, VIP, Wanfang)electronic databases, contact with authors, trial registers, or grey literature. For articles with incomplete information, corresponding authors were contacted whenever possible to obtain detailed information.

INPLASY registration number: This protocol was registered with the International Platform of Registered Systematic Review and Meta-Analysis Protocols (INPLASY) on 22 June 2021 and was last updated on 22 June 2021 (registration number INPLASY202160081).

\section{INTRODUCTION}

Review question / Objective: This systematic review aimed to comprehensively review the characteristics of DRPs for patients in primary health care institutions, which might be helpful to find effective strategies to identify, prevent and intervene with DRPs in the future.

Rationale: We searched three English databases (EMbase, The Cochrane library, PubMed) and four Chinese databases (CNKI, CBM, VIP, Wanfang). Two of 
researchers independently conducted literature screening, quality evaluation and data extraction. Qualitative and quantitative methods were combined to analyse the data.

Condition being studied: The condition of DRPs in primary health care institutions should be paid more attention, but there is a lack of systematic review to comprehensively analyze the characteristics of DRPs for patients in primary health care institutions.

\section{METHODS}

Search strategy: Computer retrieval was conducted in 3 English databases (PubMed, The Cochrane library, EMbase) and 4 Chinese databases (CNKI, CBM, VIP, Wanfang), from their inception dates to December 17, 2020. Additionally, we manually searched Google, Baidu, and the reference lists of included studies. We consulted an informatics expert and developed the search strategies which were combined subject headings and free text terms. PubMed: (("Primary Health Care"[MeSH Terms]) OR primary health care[Title/Abstract] OR (primary medical care[Title/Abstract] OR primary care[Title/ Abstract] OR Primary Healthcare[Title/ Abstract] OR community[Title/Abstract] OR communities[Title/Abstract] OR town[Title/ Abstract] OR towns[Title/Abstract] OR t ownship [ Tit le / A b s tract] OR townships[Title/Abstract] OR county[Title/ Abstract] OR counties[Title/Abstract] OR village[Title/Abstract] OR villages[Title/ Abstract] OR rural[Title/Abstract] OR ("Rural Health Services"[MeSH Terms]) OR clinic[Title/Abstract] OR clinics[Title/ Abstract])) AND (drug related problem*[Title/Abstract] OR medication related problem*[Title/Abstract] OR medicine related problem*[Title/Abstract] OR drug therapy problem*[Title/Abstract] OR medication therapy problem*[Title/ Abstract] OR medicine therapy problem*[Title/Abstract] OR therapy related problem*[Title/Abstract]). Cochrane library: \#1 MeSH descriptor: [Primary Health Care] explode all tree \#2 (Primary Health Care):ti.ab.kw \#3 (primary medical care):ti.ab.kw \#4 (primary care):ti.ab.kw \#5 (Primary Healthcare):ti.ab.kw \#6 ( com munity):ti.ab.kw \# 7 (communities):ti.ab.kw \#8 (town):ti.ab.kw \#9 (towns):ti.ab.kw \#10 (township):ti.ab.kw \#11 (townships):ti.ab.kw \#12 (county):ti.ab.kw \#13 (counties):ti.ab.kw \#14 (village):ti.ab.kw \#15 (villages):ti.ab.kw \#16 (rural):ti.ab.kw \#17 MeSH descriptor: [Rural Health Services] explode all tree \#18 (clinic):ti.ab.kw \#19 (clinics):ti.ab.kw \#20 \#1 OR \#2 OR \#3 OR \#4 OR \#5 OR \#6 OR \#7 OR \#8 OR \#9 OR \#10 OR \#11 OR \#12 OR \#13 OR \#14 OR \#15 OR \#16 OR \#17 OR \#18 OR \#19 \#21 (“drug related problem*"):ti.ab.kw \#22 ("medication related problem*"): ti.ab.kw \#23 ("medicine related problem"”): ti.ab.kw \#24 ("drug therapy problem*"):ti.ab.kw \#25 ("medication therapy problem*"):ti.ab.kw \#26 ("medicine therapy problem*"):ti.ab.kw \#27 ("therapy related problem*"):ti.ab.kw \#28 \#21 OR \#22 OR \#23 OR \#24 OR \#25 OR \#26 OR \#27 \#29 \#20 AND \#28. EMbase: \#1 primary medical care.mp. or exp primary medical care/ \#2 primary health care.mp. or exp primary health care/ \#3 primary care.mp. \#4 Primary Healthcare.mp. \#5 community.mp. or exp community/ \#6 communities.mp. \#7 town.mp. \#8 towns.mp. \#9 township.mp. \#10 townships.mp. \#11 county.mp. \#12 counties.mp. \#13 village.mp. \#14 villages.mp. \#15 rural.mp. or exp rural hospital/ or exp rural health care/ \#16 clinic.mp. \#17 clinics.mp. \#18 drug related problem*.mp. \#19 medication related problem*.mp. \#20 medicine related problem*.mp. \#21 drug therapy problem*.mp. \#22 medication therapy problem*.mp. \#23 medicine therapy problem*.mp. \#24 therapy related problem*.mp. \#25 1 or 2 or 3 or 4 or 5 or 6 or 7 or 8 or 9 or 10 or 11 or 12 or 13 or 14 or 15 or 16 or 17 \#26 18 or 19 or 20 or 21 or 22 or 23 or 24 \#27 25 and 26.

2.Chinese databases CNKI: 高级检索: 主 题: 用药相关问题 + 药物相关问题 + 药物治疗 相关问题 + 药物治疗问题 + 治疗相关问题 主 题：基层 + 社区 + 街道 + 乡镇 + 农村 + 卫生服 务中心 + 卫生院 + 卫生室 + 门诊部 + 诊所 CBM: ("初级卫生保健"[不加权:扩展] OR "初级 卫生保健"[常用字段:智能] OR "乡村卫生服务 
"[不加权:扩展] OR "乡村卫生服务"[常用字段: 智能] OR "基层"[常用字段:智能] OR "社区"[常 用字段:智能] OR "街道"[常用字段:智能] OR " 乡镇"[常用字段:智能] OR "农村 OR"[常用字段: 智能] OR "卫生服务中心"[常用字段:智能] OR " 卫生院"[常用字段:智能] OR "卫生室"[常用字 段:智能] OR "门诊部"[常用字段:智能] OR "诊 所"[常用字段:智能]) AND ("用药相关问题"[常 用字段:智能] OR "药物相关问题"[常用字段:智 能] OR "药物治疗相关问题"[常用字段:智能] OR "药物治疗问题"[常用字段:智能] OR “治疗 相关问题”[常用字段:智能]). VIP: ( $M=$ 用药相关 问题 OR R=用药相关问题 OR $M=$ 药物相关问 题 OR $R=$ 药物相关问题 OR $M=$ 药物治疗相关 问题 OR $R=$ 药物治疗相关问题 OR $M=$ 药物治 疗问题 OR R=药物治疗问题 OR $\mathbf{M}=$ 治疗相关 问题 OR $R=$ 治疗相关问题) AND ( $M=$ 基层 OR $R=$ 基层 OR $M=$ 社区 OR $R=$ 社区 OR $M=$ 街道 OR $R=$ 街道 OR $M=$ 乡镇 OR $R=$ 乡镇 OR $M=$ 农 村 OR $R=$ 农村 OR $M=$ 卫生服务中心 OR $R=$ 卫 生服务中心 OR M=卫生院 OR $R=$ 卫生院 OR $M=$ 卫生室 OR $R=$ 卫生室 OR $M=门$ 门诊部 OR $R=$ 门诊部 OR $M=$ 诊所 OR $R=$ 诊所) WanFang: 专业检索：(主题:("用药相关问题") or 主题:(" 药物相关问题") or 主题:("药物治疗相关问题" or 主题:("药物治疗问题") or 主题:("治疗相关问 题")) and (主题:("基层") or 主题:("社区") or 主 题:("街道") or 主题:("乡镇") or 主题:("农村") or 主题:("卫生服务中心") or 主题:("卫生院") or 主 题:("卫生室") or 主题:("门诊部") or 主题:(“诊所 ")).

Participant or population: Patients visiting primary health care institutions, including community health service centers, community health service stations, street health centers, township health centers, village health offices, outpatient departments and clinics (infirmaries).

Intervention: With or without intervention.

Comparator: With or without comparator.

Study designs to be included: The types of study design were not limited.
Eligibility criteria: Inclusion criteria: (i) Patients visiting primary health care institutions, including community health service centers, community health service stations, street health centers, township health centers, village health offices, outpatient departments and clinics (infirmaries) (National Health And Family Planning Commission, 2020); (ii) Relevant studies reporting the characteristics of DRPs, including the incidence, types, causes, risk factors, and the acceptability of interventions. Exclusion criteria: (i) Duplicate publications; (ii) Articles which wasn't in English or Chinese; (iii) Articles without an available full text. The types of study design were not limited as the aim of this research was to conduct a comprehensive review of all published studies.

Information sources: 3 English databases (PubMed, The Cochrane library, EMbase) and 4 Chinese databases (CNKI, CBM, VIP, Wanfang) electronic databases, contact with authors, trial registers, or grey literature3 English databases (PubMed, The Cochrane library, EMbase) and 4 Chinese databases (CNKI, CBM, VIP, Wanfang) electronic databases, contact with authors, trial registers, or grey literature. For articles with incomplete information, corresponding authors were contacted whenever possible to obtain detailed information.

Main outcome(s): The incidence of DRPs, the types of DRPs, the causes of DRPs, the risk factors of DRPs.

Additional outcome(s): The acceptability of DRPs interventions.

Quality assessment / Risk of bias analysis: Currently, there were no more accepted quality assessment instruments for crosssectional studies (Sanderson et al., 2007). After discussions, we decided to choose a relatively widely used scale, the Agency for Healthcare Research and Quality scale. Because we only focused on the preintervention phase, the quality of intervention studies also were evaluated by the Agency for Healthcare Research and 
Quality scale. Two researchers (XF Ni and YM Bai) independently evaluated the quality of the included articles using the AHRQ scale with 11 items, each of which was answered with "yes", "no" and "unclear". Any disagreements after crosschecking were resolved by discussions between the two researchers with the final decisions of the third researcher (CS Yang). If the answer was "no", "unclear" or "not applicable", the item was given a score of " 0 "; if the answer was 'yes', the item was scored as " 1 ". The quality assessments of the articles were classified as follows: low quality $=0-3$, medium quality $=4-7$, high quality = 8-11 (Hu et al., 2015; Zeng et al., 2015).

Strategy of data synthesis: A combination of qualitative and quantitative analysis was used in this research. If the initial data was not expressed as the mean and standard deviation, the sample size, median, range and/or inter-quartile range (IQR) were used to estimated the mean and standard deviation for comparison purposes (Luo et al., 2018; Wan et al., 2014). As different included studies chose different DPRs classification systems, we reclassified the types and cause of DPRs according to the PCNE Classification for Drug-Related Problems Version 9.1 (Plácido et al., 2020); DRPs were only be classified in the main domain when there was not enough information to make a clear classification in a particular subdomain.

Subgroup analysis: None.

Sensitivity analysis: None.

Language: English or Chinese.

Country(ies) involved: China.

Keywords: Drug-Related Problems, Primary Heath Care, Systematic Review, Medication Review, Pharmaceutical Service.

Contributions of each author:

Author 1 - Xiaofeng Ni.

Author 2 - Chunsong Yang.

Author 3 - Yumei Bai.

Author 4 - Zixian Hu.

Author 5 - Lingli Zhang. 